\title{
Breakdown of the ponderomotive approximation as an acceleration mechanism in wave-particle nonlinear dynamics
}

S. Marini, E. Peter, G. I. de Oliveira, and F. B. Rizzato

Citation: Physics of Plasmas 24, 093113 (2017); doi: 10.1063/1.4995524

View online: https://doi.org/10.1063/1.4995524

View Table of Contents: http://aip.scitation.org/toc/php/24/9

Published by the American Institute of Physics

\section{Articles you may be interested in}

Simulations of a beam-driven plasma antenna in the regime of plasma transparency

Physics of Plasmas 24, 093114 (2017); 10.1063/1.4995323

Electron holes in phase space: What they are and why they matter

Physics of Plasmas 24, 055601 (2017); 10.1063/1.4976854

Triplet and beam interaction in a plasma

Physics of Plasmas 24, 102124 (2017); 10.1063/1.5005946

Ultrafast multi-MeV gamma-ray beam produced by laser-accelerated electrons

Physics of Plasmas 24, 093104 (2017); 10.1063/1.4996020

Astrophysical particle acceleration mechanisms in colliding magnetized laser-produced plasmas Physics of Plasmas 24, 092901 (2017); 10.1063/1.4993204

Formation of electron energy spectra during magnetic reconnection in laser-produced plasma

Physics of Plasmas 24, 102101 (2017); 10.1063/1.4994260

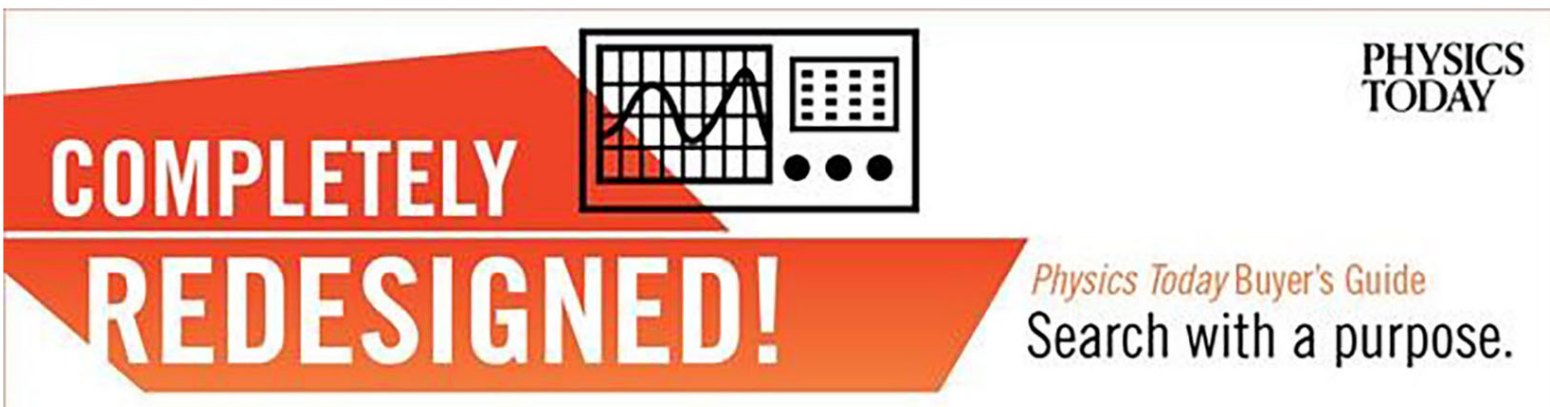




\title{
Breakdown of the ponderomotive approximation as an acceleration mechanism in wave-particle nonlinear dynamics
}

\author{
S. Marini, ${ }^{1, a)}$ E. Peter, ${ }^{1, b)}$ G. I. de Oliveira, ${ }^{2, c)}$ and F. B. Rizzato ${ }^{1, d)}$ \\ ${ }_{1}^{1}$ Instituto de Física, Universidade Federal do Rio Grande do Sul, Caixa Postal 15051, \\ 91501-970 Porto Alegre, RS, Brasil \\ ${ }^{2}$ Instituto de Física, Universidade Federal do Mato Grosso do Sul, Caixa Postal 549, \\ 79070-900 Campo Grande, MS, Brasil
}

(Received 12 July 2017; accepted 18 August 2017; published online 8 September 2017)

\begin{abstract}
In the present analysis, we study the dynamics of charged particles submitted to the action of slowly modulated electromagnetic carrier waves. While the velocity of the particles remains smaller than the carrier's phase-velocity, their dynamics is well described by a refined ponderomotive approach. The ponderomotive approach has its own validity limits well established, beyond which particles are resonantly trapped by the carrier waves. We show that under adequate conditions, the trapping mechanism places particles at an optimal relative phase with respect to the carrier for maximum acceleration. In addition to the analytical approach involved in the ponderomotive description, we use numerical simulations to validate the corresponding dynamics as well as to explore various features of the resonant trapping and acceleration. Published by AIP Publishing.
\end{abstract}

[http://dx.doi.org/10.1063/1.4995524]

\section{INTRODUCTION}

The ponderomotive approximation for the nonlinear dynamics of particles in high-frequency fields attempts to describe the full dynamical problem in terms of a selfconsistent set of time averaged, low-frequency variables. ${ }^{1}$ The procedure leading to the approximation is valid as long as particles are not in resonance with the driving waves. What is typically left after high-frequency components of the dynamics are removed is an energy conserving system, provided no dependence on slow time scales remains. The occurrence of energy conservation in ponderomotive regimes simplifies the description of the dynamical system, but prevents particles from a net energy gain when these particles interact with localized steady state envelopes of highfrequency waves. Under this condition, the kinetic energy of particles before and after the interaction would be the same.

On the other end of the spectrum of dynamical regimes, one finds the resonant regimes. Here particles can be highly accelerated as they move in phase with the high frequency carriers, as for instance is the case of particle acceleration by self-sustained electromagnetic (EM) waves in plasmas. ${ }^{2,3} \mathrm{~A}$ classic example is the beat-wave accelerator scheme, where a coherent electrostatic plasma wave is nonlinearly generated by the beating of transverse laser modes and particles are resonantly accelerated as they are injected with the proper resonant phase with respect to the electrostatic mode. ${ }^{4}$

The beat wave accelerator evolved into a large number of sophisticated variants. In current experiments, the preferred profiles for the driver structures are based either on localized laser modes or on bunched beams of charged particles, ${ }^{5}$ both

\footnotetext{
a)marini@ufrgs.br

b) peterpeter@uol.com.br

c)glaucius.oliveira@ufms.br

d)rizzato@if.ufrgs.br
}

of which can provide higher field intensities in the region of interest. ${ }^{6,7}$ What remains as a needed crucial condition in all variants is the adjustment of a proper resonant phase between accelerating fields and particles. ${ }^{2,8}$

Proper wave-particle tuning demands fine control of the beam-injection process, ${ }^{9-11}$ so one might be interested in settings where tuning can be achieved automatically by the own system, without any extra intervention of the experimenter. One way to do that is to use intense electromagnetic waves in neutral gases - the gas is ionized and the resulting charged particles are self-adjustably accelerated by the waves. ${ }^{12,13}$ In the present work, we develop a ponderomotive formalism to discuss the possibility of efficient acceleration within the context of the self-adjusted phase. The ponderomotive approach enters as a crucial tool to that effect.

In the model, a high-frequency electrostatic wave is smoothly modulated along its wavevector by a steady-state envelope which has a length scale much larger than the wavelength of the carrier. A relativistic particle is then injected along the wavevector axis and allowed to approach the region occupied by the envelope with smaller velocity than the carrier's phase-speed.

Under conventional ponderomotive conditions ${ }^{14,15}$ where particles see a very high Doppler-shifted carrier frequency, ${ }^{16,17}$ the model becomes integrable and these particles either advance across or are reflected off the envelope region with no net energy gain, as mentioned earlier. The present work discusses the possibility that the initially nonresonant particle lagging the carrier can still be highly accelerated as it emerges from the envelope region. This involves an initial ponderomotive stage followed by trapping by the rising levels of the envelope potential field seen by the moving particle and, through an autonomic phase adjusting, a subsequent catapulting acceleration as particles go all the way downhill from the potential peak just after capture in a wave trough. In a nutshell, if 
particles are injected with smaller velocities than the wave phase-velocity, they keep slipping backwards relatively to the wave up to a point where the wave crests may become sufficiently large that end up like a potential wall pushing particles forwardly. We point out that trapping occurs when the full particle's velocity, not only its average speed, becomes resonant with the carrier's speed. We therefore require a refined ponderomotive calculation that allows to obtain not only average, but also peak oscillatory velocities.

\section{THE PONDEROMOTIVE FORMALISM}

In our model, we examine the interaction of a relativistic particle with an electrostatic modulated wave of the form

$$
\varphi(x, t)=\varphi_{0} e^{-\frac{x^{2}}{\sigma^{2}}} \cos (k x-\omega t) .
$$

The amplitude $\varphi_{0}$ is a constant, $k$ and $\omega$ are the fast wavevector and frequency of a carrier moving along the $x$ axis, and $\sigma$ measures the envelope length; we assume $\sigma \gg 1 / k$ to enforce the condition of a slowly modulated wave train.

From the fully relativistic Lagrangian of a particle with charge $q$ and mass $m$

$$
L=-m c^{2} \sqrt{1-\dot{x}^{2} / c^{2}}-q \varphi(x, t),
$$

a canonical Hamiltonian can be obtained in the following dimensionless form

$$
H=\gamma+\varphi_{0} e^{-x^{2} / \sigma^{2}} \cos (x-t),
$$

where space and time are normalized by $k^{-1}$ and $\omega^{-1}$, respectively, $\gamma=\sqrt{1+p^{2} / \alpha}$ is the relativistic factor with $p$ as the dimensionless momentum, $\alpha \equiv v_{\phi}^{2} / c^{2}$ with $v_{\phi}=\omega / k$ as the phase-velocity of the carrier, and $c$ the speed of light. In addition, $H\left(\varphi_{0}\right)$ is normalized by the factor $m c^{2}\left(m c^{2} / q\right)$. Mass and charge are kept arbitrary at this point, but occasionally along the text we discuss applications to electron acceleration. We point out that while the velocity is normalized by $v_{\phi}$, the momentum is normalized by $m c^{2} / v_{\phi}$.

We point out that even though our relativistic model is purely electrostatic, as long as the particle dynamics parallel to the wavevector axis is concerned, the underlying physics is similar to that of a particle submitted to the combined action of collinear electromagnetic and wiggler fields. If either one is modulated and both are sufficiently intense, the resulting force field due to this inverse free-electron laser arrangement ${ }^{18-20}$ would be similar to that of the purely potential field. Note that while the generation of coherent plasma waves involving plasma or neutral gas environments may be a complicated task from the experimental point of view, the vacuum electronics behind the free-electron laser scheme may be more easily achieved in laboratory.

As mentioned earlier, we know what happens in ideal ponderomotive conditions and we know what happens exactly at resonance. In the present work, we will be interested in the system performance as one gradually moves from ideal ponderomotive conditions towards resonance. It is in this crossover region that the optimum trapping mechanism of acceleration discussed earlier takes place.
We therefore begin with the analysis looking for a ponderomotive approximation for the full model represented by Eq. (3). The conventional ponderomotive approximation demands that one is far away from resonance, the latter written as $p /(\alpha \gamma)-1=0$ in our dimensionless system, and that the envelope is long enough that $\sigma \gg 1$. Under these conditions, the local particle dynamics is slightly affected by the highfrequency carrier and one searches for the average secular dynamics of the particle's oscillation centre.

Following the formal norm of Hamiltonian perturbations, one performs a canonical transformation that removes the high-frequency components of Hamiltonian (3). We consider a generating function $F(x, P)=x P+f(x, P, t)$ depending on the old coordinate $x$ and on the new momentum $P$. The relationship between old and new momenta reads $p=P+\partial f / \partial x$ and we look for the function $f$ that renders $P$ a low-frequency variable. In other words, we construct $f$ such that it absorbs all the high-frequency jittering of the dynamics.

We initially suppose to be sufficiently far from resonances that the high frequency jitter is small, which allows to expand $\gamma$ in Eq. (3) in the form

$$
\gamma \approx \Gamma+\frac{P \partial f / \partial x}{\alpha \Gamma}+\frac{(\partial f / \partial x)^{2}\left(1-\frac{P^{2}}{\alpha \Gamma^{2}}\right)}{2 \alpha \Gamma},
$$

where $\Gamma \equiv \sqrt{1+P^{2} / \alpha}$. Under the canonical transformation, the original Hamiltonian is changed into $h=H+\partial f / \partial t$ and if one requires that the linear terms on the derivatives of $f$ of the newly formed Hamiltonian sum up to cancel the potential term up to first order, $f$ can be found in the form

$$
(f)_{1^{s t} \text { order }}=\varphi_{0} e^{-x^{2} / \sigma^{2}} \sin (x-t) /[P /(\alpha \Gamma)-1] .
$$

In terms of order of magnitude, one sees that $f$ goes with the inverse of the Doppler shifted frequency as mentioned earlier.

Expression (5) can be used to evaluate the squared term involving $\partial f / \partial x$ which can be separated into high and lowfrequency components. The high-frequency part can then be removed with a second-order canonical transformation. If one stops at this first cycle, the resulting ponderomotive Hamiltonian reads

$$
H=\Gamma+\frac{1}{4 \alpha \Gamma} \frac{\varphi_{0}^{2} e^{-2 X^{2} / \sigma^{2}}}{(P /(\alpha \Gamma)-1)^{2}}\left(1-\frac{P^{2}}{\alpha \Gamma^{2}}\right),
$$

where the capital $X$ represents the oscillation center position. Note that variable $x$ can be replaced with $X$ in the exponential owing to the fact that the difference between these two variables is much smaller than the envelope length scale under the ponderomotive approximation. As for the generating function correct up to the current order of approximation, one has

$$
\begin{aligned}
f= & \frac{\varphi_{0} e^{-X^{2} / \sigma^{2}} \sin (x-t)}{P /(\alpha \Gamma)-1} \\
& -\frac{\varphi_{0}^{2} e^{-2 X^{2} / \sigma^{2}} \sin [2(x-t)]}{8(P /(\alpha \Gamma)-1)^{3}}\left(1-\frac{P^{2}}{\alpha \Gamma^{2}}\right) .
\end{aligned}
$$


At this point we illustrate the accuracy of our model, by comparing the full dynamics generated either by Eq. (2) or by the corresponding full Hamiltonian (3), with the ponderomotive approximation as represented by Hamiltonian (6) and the corresponding generating function (7).

For given values of $\alpha$ and $\varphi_{0}$, in Fig. 1 we examine two typical cases: the case of reflected particles which occurs at small enough injection velocities $v_{0}$ of panel (a) and the case of passing particles which occurs at large enough velocities of panel (b). We start our numerical essays with $\alpha^{1 / 2}=0.9$ since this provides a solid view on the general aspects of the problem. Extensions to higher values of $\alpha$ are important, have critical implication, and will be discussed later on.

In the two panels of Fig. 1 we consider $\varphi_{0}=0.5$, with $v_{0}=0.3 \alpha^{-1 / 2}$ in (a) and $v_{0}=0.5 \alpha^{-1 / 2}$ in (b) (note that under the present dimensionless scalings, $v \alpha^{1 / 2}$ is precisely the ratio of the dimensional velocity to the speed of light, $v_{\mathrm{dim}} / \mathrm{c}$ ); particles are always injected from $x(t=0)=-5 \sigma$ although all results in the paper are independent of any initial wave-particle relative phase. In both cases of Fig. 1, the full solutions (in red) obtained from the complete Lagrangian (2) are superposed with thick dashed lines representing the enveloping velocities associated with the momenta $P+\partial f / \partial x$ calculated from the ponderomotive approximation. The upper (lower) enveloping curve is computed as the carrier's phase term $\sin (x-t)$ is replaced with its maximum (minimum) excursion value +1 $(-1)$. One observes that the agreement provided by the present ponderomotive theory is truly satisfactory.

\section{BREAKDOWN OF THE PONDEROMOTIVE APPROXIMATION AND ACCELERATION}

We now proceed to the main point of this work and see what happens when the peaks of velocity excursions reach the resonant condition. At this point particles are captured by the potential well and can be accelerated towards $c$ as much as the amplitude $\varphi_{0}$ and proper initial phase allow.

Once particles are trapped by the carrier, one can relate $\varphi_{0}$ to the maximum possible velocity if the particle goes downhill from the maximum to the minimum of the potential. An estimate reads

$$
\varphi_{0} \sim[(1-\sqrt{\alpha})] /\left[2 \sqrt{2} \sqrt{1-v_{f, \operatorname{dim}} / c}\right],
$$
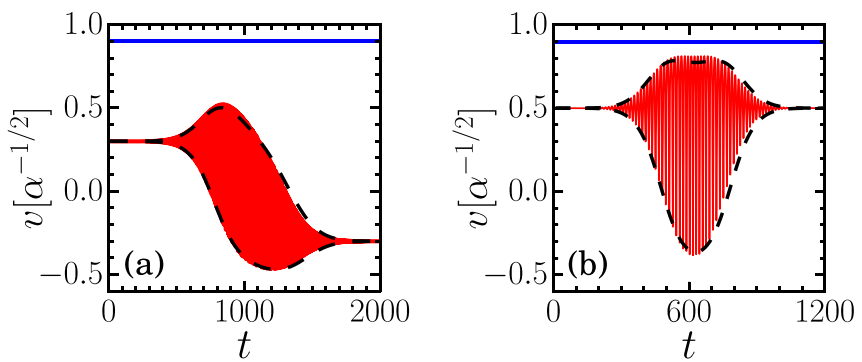

FIG. 1. Evolution of particle velocity for $\sqrt{\alpha}=0.9, \varphi_{0}=0.5$, and $\sigma=100$. Reflected particles in (a) with $v_{0}=0.3 \alpha^{-1 / 2}$ and passing particles in (b) with $v_{0}=0.5 \alpha^{-1 / 2}$. The blue solid line represents the resonant velocity $v_{\phi, d i m} / c$ $=\alpha^{1 / 2}$, while the dashed black lines are obtained from the ponderomotive approximation. The peaks of velocity do not reach the resonant condition in either case. from which one observes that with amplitudes around $\varphi_{0}$ $\sim 0.5$ as in the previous figures, final velocities on the order of $v_{f, d i m} / c \geqslant 0.99$ can be attained when $\alpha^{1 / 2}=0.9$. The estimate can be obtained if one takes Hamiltonian (3) and neglects the slow modulation for the fast resonant energy gain. Under this condition, one has a purely harmonic wave of amplitude $\varphi_{0}$ to which one associates a conserved quantity $H-p$ obtained from canonical expressions $d H / d t=\partial H / \partial t$ and $d p / d t=-\partial H / \partial x$. Next, if one inputs a particle with the resonant phase velocity at the peak of the cosine potential as the initial condition, the velocity at the bottom can be obtained as a function of $\varphi_{0}$ through the conserved quantity. Expression (9) is finally obtained under the approximation $\left(c-v_{f, \text { dim }}\right) / c \ll 1$, valid for fast particles.

In our next figure, Fig. 2, we examine a trapping event. A peak in the ponderomotive oscillation is about to cross the resonant condition in panel (a), the peak occurring at the maximum of the modulated field at $x=0$ owing to the symmetry. Particles still lag the wave. For a slightly larger value of $v_{0}$, the peak crosses the resonant line and the particle is catapulted forward as it is pushed by a maximum of the potential well. We point out that the particle is not pushed back by the next potential maximum because as the particle advances, the modulation reduces the maximum height of the potential wave and allows barrier crossing. Note that forwardly directed extraction at maximum speed is then inherent to the mechanism discussed here.

Figure 3 offers a global view of the ponderomotive dynamics and resonant acceleration just examined.

In the 2D plot of the figure, we depict the colour graded final velocity $v_{f}$ that particles injected with velocity $v_{0}$ exit the accelerating region occupied by a modulated wave mode of maximum amplitude $\varphi_{0}$. The accelerating region resulting from resonant trapping is seen in vivid red/yellow colors. The bounding curve indicating the onset of the trapping mechanism is obtained from the model and once again we see that simulations agree well with the analytical estimates. The more subdued colors indicate regions where the ponderomotive approximation is valid. The upper region, that of higher $\varphi_{0}$ 's, corresponds to particles reflected off the potential hump, while the lower region corresponds to passing particles. There is a frontier between both regions, the locus of which again agrees with the dotted curve obtained by our approximate model. Finally, the four cases studied in
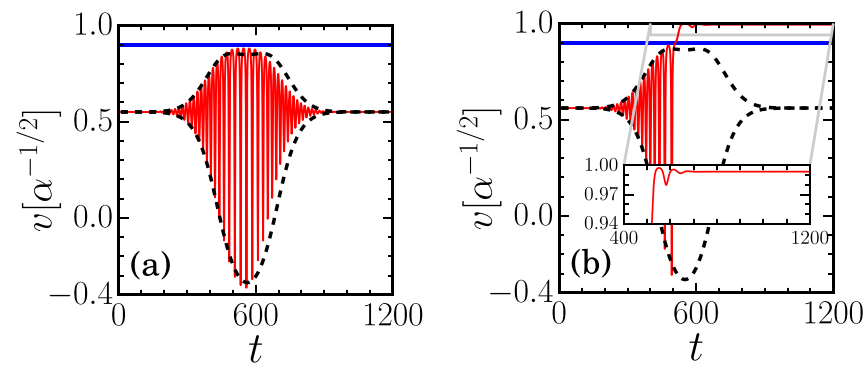

FIG. 2. Same as in Fig. 1: $v_{0}=0.55 \alpha^{-1 / 2}$ in (a) and $v_{0}=0.60 \alpha^{-1 / 2}$ in (b). In panel (a), the peak of velocity is slightly below the resonant wave's phase-velocity. In panel (b), the peak of velocity crosses the resonance line, ponderomotive dynamics breaks down, and the particle is accelerated towards $c$ as detailed in the inset. 


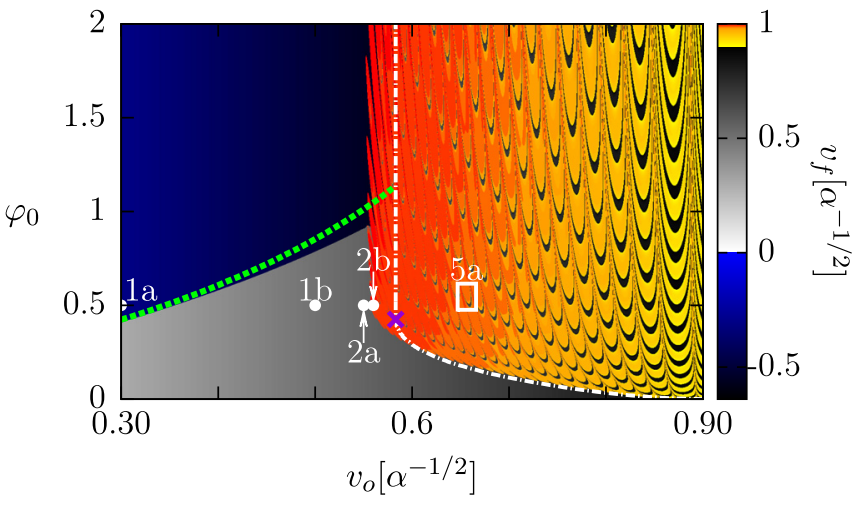

FIG. 3. Color graded map of the exiting speed $v_{f}$ for $\alpha^{1 / 2}=0.9$ and $\sigma=$ 100. Gray (blue) hues represent the reflective (passing) regime, yellow roughly indicates velocities between $0.9 c$ and $0.99 c$, and red indicates velocities a bit greater than $0.99 c$. The green and white dashed lines indicate the ponderomotive approximations for the reflective/passing frontier and for the boundary of the resonant regime. The points and region shown in details in other figures are marked and labeled in the map.

Figs. 1 and 2 are marked accordingly. The trapping event analysed in Fig. 2 was chosen to occur around the left corner of the trapping region. The corner is defined as the point along the trapping boundary where the ponderomotive approximation first develops a double hump profile preceding the curve bending and the reflective profile. The corner is indicated as a cross in the plot and Fig. 2(a) shows the double hump forming. The corner region is the one where, with the lowest field amplitudes, one achieves the maximum predicted final velocity starting with the lowest injected velocity. Accelerating efficiency does not change much as $\varphi_{0}$ grows and actually diminishes as the injection velocity becomes larger, so the corner is the optimum region for acceleration. We shall return to these topics in a moment.

\section{A. Choice of $\alpha$}

As mentioned earlier, this may be the appropriate occasion to discuss the choice and role of $\alpha$ on the accelerating mechanism. What happens is that as $\alpha$ decreases, the lefthand side corner of the trapping region in Fig. 3 scales down to smaller values of $\varphi_{0}$ and $v_{0}$. At the same time, relation (8) shows that for a given exiting velocity, $\varphi_{0}$ increases from zero when $\alpha$ decreases. There is thus a unique value of $\alpha$ where the amplitude $\varphi_{0}$ at the corner coincides with the needed amplitude for a given final ejection velocity. The value of $\alpha$ corresponding to final velocities of the order of $99 \%$ of $c$, sits around $\alpha=0.78$, which is nearly what we use in our first numerical discussion. This is seen in Fig. 4 where we plot the amplitude at the corner $\left(\varphi_{0, c o r}\right)$ and the amplitude for resonant acceleration $\left(\varphi_{0, \text { res }}\right)$ as obtained from Eq. (8) for a variety of final velocities. As indicated in Fig. 4, larger values of $\alpha$ led to increasingly larger values of the final velocity as well. One should notice that at the largest illustrated $\alpha$ in the plot, even for a moderate value of $\varphi_{0}$ the final electronic energies - in the case of electron acceleration-approach the scale of GeV's from relatively small injection velocities around $0.7 c$.

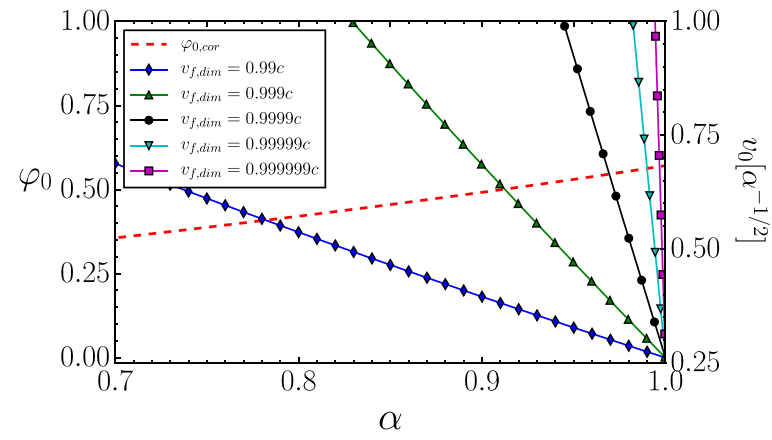

FIG. 4. Intersection of curves $\varphi_{0, \text { cor }}$ and $\varphi_{0, \text { res }}$ indicating the ideal operating point for various final velocities $v_{f, d i m}$. On the right vertical axis we represent the corresponding injection velocities $v_{0}$ at the corner.

\section{B. Optimum acceleration at the corner}

Finally, why is the corner optimum? One notices a roughly periodic array of less-than-effective discrete curves inside the accelerating region of Fig. 3. We have examined these singular curves and found out that their vertices correspond to "fixed points" of the dynamics in the velocity space, where exiting and injecting velocities are identical. As one moves along the wings emanating from the vertices, both velocities become different, but still with the exiting speed below resonance. A detailed view of the dynamics at the vertice, highlighted in Fig. 3, is seen in Fig. 5. The feature is not dissimilar to what is seen in parametric analysis of bifurcating dissipative systems. ${ }^{21}$

In addition, the number of oscillations within the resonant region increases by one unit each time one crosses upwards/ rightwards each layer of (almost) side-by-side vertices. This feature causes the dynamics to increasingly resemble an adiabatic one as one tries to augment either the injected velocity $v_{0}$ or the amplitude $\varphi_{0}$ with a view on a possible improvement of the accelerating process. It appears that in both circumstances particles are simply captured at lower levels of the modulated potential before reaching the peak, execute a large number of action-conserving oscillations inside the slowly varying potential well, and are gently released at lower velocities than the maximum.

\section{CONCLUSION}

In summary, we have studied the breakdown process of the ponderomotive dynamics of a particle in the field of a smoothly varying EM wave. In the present analysis, the EM wave is modeled after an electrostatic mode, but one expects
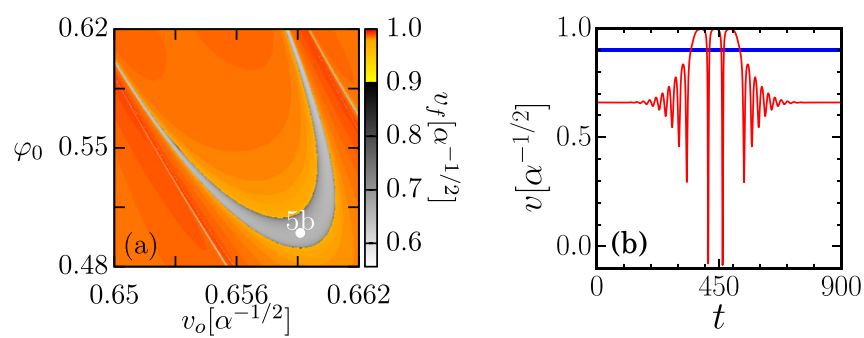

FIG. 5. Panel (a) expands the corresponding region of Fig. 3 and panel (b) explores in detail the dynamics of the fixed point at $v_{0}=0.65913 \alpha^{-1 / 2}$ and $\varphi_{0}=0.49988$. 
the same kind of behaviour if the longitudinal forces of the electrostatic mode are replaced with those generated by the beating of collinear laser and wiggler fields in an inverse free-electron laser process, for instance.

Dynamics is well described by ponderomotive approximations as long as one stays away from wave-particle resonance. If one increases either the injection velocity or the field amplitude $\varphi_{0}$ such that resonance is attained, particles can be highly accelerated under adequate conditions. These adequate conditions are generically represented by the red/ yellow regions of the color graded plot of Fig. 3. However, as just discussed, efficiency is much higher if one works close to the left-hand-side corner where particles are catapulted instead of undergoing the adiabatic-like process where several oscillations take place while the particle remains trapped.

Overall efficiency is magnified if $\alpha$ is such that the value of $\varphi_{0}$ at the left corner of the resonant region coincides with the needed amplitude to make particles leap from the resonant velocity to the vicinity of the speed of light. For $v_{f, \operatorname{dim}} / c \gtrsim 0.99$, these two threshold amplitudes become similar when $\alpha \sim 0.78$, which requires usage of only moderately relativistic amplitudes on the order of $\varphi_{0} \sim 0.4-0.5$. The acceleration process is nevertheless very efficient because it is not based on the strength of the wave amplitude alone, but also on the automatic positioning of particles at the peak of the wave field, which is provided by the synergetic ponderomotive process. The choice of larger values of $\alpha$ leads to increasingly larger final energies.

The present model does not take into account multidimensional aspects associated with the wave-particle interaction: inclusion of misalignment between the incoming particles and the carrier's wavevector as well as the finite cross section of a real accelerating field, for instance, may reduce the acceleration efficiency. We have also modeled the wave field in terms of a pure electrostatic mode, which is somewhat equivalent but not equal to the inverse free-electron laser discussed earlier. In addition, given the large accelerations particles are submitted to, another important feature to be investigated is the problem of radiation reaction on particles and the self-consistent dynamics of the accelerating fields in case one works with relatively dense particle beams.

Further study is needed on the issues of the previous paragraph, but despite its shortcomings we still expect the present 1D analysis to be adequate as a first approach to understand the underlying nonlinear dynamics of the full process.

\section{ACKNOWLEDGMENTS}

We acknowledge support from $\mathrm{CNPq}$, Brasil and from AFOSR, USA, under research Grant No. FA9550-16-1-0280.

${ }^{1}$ P. Mulser and D. Bauer, High-Power Laser-Matter Interaction (SpringerVerlag, Berlin, 2010).

${ }^{2}$ P. K. Shukla, N. N. Rao, M. Y. Yu, and N. L. Tsintsadze, Phys. Rep. 138, 1 (1986).

${ }^{3}$ T. M. C. da Silva, R. Pakter, F. B. Rizzato, M. C. de Sousa, I. L. Caldas, and F. M. Steffens, Phys. Rev. E 88, 013101 (2013).

${ }^{4}$ T. Tajima and J. M. Dawson, Phys. Rev. Lett. 43, 267 (1979).

${ }^{5}$ E. Esarey, C. B. Schroeder, and W. P. Leemans, Rev. Mod. Phys. 81, 1229 (2009).

${ }^{6}$ D. Farina and S. V. Bulanov, Phys. Rev. Lett. 86, 5289 (2001).

${ }^{7}$ R. Bingham, Nature 424, 258 (2003).

${ }^{8} \mathrm{~J}$. T. Mendonça, Theory of Photon Acceleration (IOP Publishing, Bristol, 2001).

${ }^{9}$ E. G. Evstatiev, P. J. Morrison, and W. Horton, Phys. Plasmas 12, 072108 (2005).

${ }^{10}$ M. Tzoufras, W. Lu, F. S. Tsung, C. Huang, W. B. Mori, T. Katsouleas, J. Vieira, R. A. Fonseca, and L. O. Silva, Phys. Rev. Lett. 101, 145002 (2008).

${ }^{11}$ K. K. Magesh Kumar and V. K. Tripathi, Laser Part. Beams 30, 575 (2012).

${ }^{12}$ S. M. Hooker, Nat. Photonics 7, 775 (2013).

${ }^{13}$ X. Zhang, V. N. Khudik, and G. Shvets, Phys. Rev. Lett. 114, 184801 (2015).

${ }^{14}$ J. R. Cary and A. N. Kaufman, Phys. Fluids 24, 1238 (1981).

${ }^{15}$ C. Grebogi and R. G. Littlejohn, Phys. Fluids 27, 1996 (1984).

${ }^{16}$ A. Macchi, A Superintense Laser-Plasma Interaction Theory Primer (Springer, London, 2013).

${ }^{17}$ D. A. Burton, R. A. Cairns, B. Ersfeld, A. Noble, S. Yoffe, and D. A. Jaroszynski, "Observations on the ponderomotive force," Proc. SPIE 10234, 102340G (2017).

${ }^{18}$ A. van Steenbergen, J. Gallardo, J. Sandweiss, and J.-M. Fang, Phys. Rev. Lett. 77, 2690 (1996).

${ }^{19}$ L. F. Monteiro, A. Serbeto, K. H. Tsui, J. T. Mendonça, and R. M. O. Galvão, Phys. Plasmas 20, 073101 (2013).

${ }^{20}$ E. Peter, A. Endler, and F. B. Rizzato, Phys. Plasmas 21, 113104 (2014)

${ }^{21}$ J. A. C. Gallas, Phys. Rev. Lett. 70, 2714 (1993). 